\title{
Kinderspitäler leiden an systematischer Untertarifierung
}

\section{Agnes Genewein}

Dr. med., MBA, MAE und FMH Pädiatrie, Schwerpunkt Neonatologie, Geschäftsführerin AllKidS

Die Kinder- und Jugendmedizin fristet in der breit gefächerten Gesundheitsversorgung oft ein Mauerblümchendasein. Das mag daran liegen, dass die 20 Prozent unserer Bevölkerung im Kinder- und Jugendalter nur vier Prozent der Gesundheitskosten verursachen, die Kinder- und Jugendmedizin weitgehend auf Husten, Schnupfen und Kinderkrankheiten reduziert wird und andere Fachrichtungen daher nicht nur lukrativer, sondern auch anderweitig viel interessanter und spektakulärer erscheinen. Doch der Schein trügt.

Die eigenständigen Kinderspitäler, die in der Allianz Kinderspitäler der Schweiz AllKidS ihre Interessen gemeinsam wahrnehmen, leisten eine hochspezialisierte Medizin. Zahlreiche der von ihnen behandelten Krankheiten kennt man noch kaum. So leidet eines von drei Kindern, das in einem AllKidS-Spital behandelt wird, an einer sogenannt "seltenen Krankheit». Während im umliegenden Ausland die spezialisierte Kinder- und Jugendmedizin zunehmend an Rückhalt

\section{Résumé}

Les coûts standard par cas des trois hôpitaux pédiatriques indépendants (Hôpital universitaire pédiatrique des deux Bâles, Hôpital pédiatrique de Suisse orientale à Saint-Gall et Hôpital des Enfants de Zurich) sont nettement supérieurs à ceux des cliniques générales. Polynomics a vérifié pourquoi [1] et conclut que ce n'est pas la conséquence de manques d'efficience. Les facteurs de coût sont les high-outliers plus chers que la moyenne, une palette très étendue et coûteuse de prestations (prestataires de soins finaux) et l'attribution de cas particulièrement complexes et sévères, parmi lesquels des enfants atteints d'infirmités congénitales et notamment des nouveau-nés malades. Le groupe de diagnostic des nouveau-nés (MDC 15), qui représente les nouveau-nés en bonne santé et malades, est littéralement fatal aux hôpitaux pédiatriques pour enfants. De faibles nombres de cas et le floutage des diagnostics par le système de classification internationale ( $\mathrm{CIM}-10)$ compromettent la rémunération adéquate des hôpitaux pédiatriques pour le travail fourni. L'intervention actuelle du Conseil fédéral dans le TARMED aggrave encore leur situation. Si la médecine spécialisée pour enfants et adolescents en Suisse doit rester au niveau que l'on considère comme allant de soi dans la médecine pour adultes, des adaptations tarifaires à différents égards sont impératives. verliert [2], sind wir in der Schweiz noch ein Stück weit davon entfernt.

Tatsache ist jedoch, dass die Kinder- und Jugendmedizin auch in unserem Land immer mehr unter Druck gerät, weil der Aufwand nicht mehr sachgerecht bzw. kostendeckend entschädigt wird. In gemischten Spitälern leistet man sich Kinder- und Jugendabteilungen noch, weil die Defizite durch Erträge anderer Fachrichtungen kompensiert werden können und die Gesamtrechnung für ein Spital am Ende doch noch aufgeht. Den drei einzigen in der Schweiz verbliebenen Spitälern, die auf die Versorgung von Kindern und Jugendlichen spezialisiert sind und dabei internationale Spitzenleistungen erbringen, ist diese Möglichkeit verwehrt. Sie müssen vollständig von dem leben, was SwissDRG und TARMED bzw. die Versicherer für Kinder- und Jugendmedizin hergeben - und das wird immer weniger. Zumal auch kaum Erträge aus Zusatzversicherungen anfallen, die Spitäler finanziell über Wasser zu halten vermögen. Kinder sind in der Regel nur grundversichert. Kinder mit einem Geburtsgebrechen werden von Versicherern nicht in eine Zusatzversicherung aufgenommen.

Bereits bei Einführung der stationären Fallpauschalen war klar, dass im Bereich der Tarife für die Kinderund Jugendmedizin Handlungsbedarf besteht. Um der Situation als Endversorgerspital gerecht zu werden, erhielten zwar auch die AllKidS-Spitäler sowie die Universitätskliniken eine höhere Baserate als Spitäler ohne Endversorgerstatus. Doch die Tarife waren von Anfang an knapp bemessen, um die Spitäler zu mehr Effizienz anzuhalten. Aus diesem Grund sollten sich die fixen Fallpauschalen an den effizient arbeitenden Spitälern orientieren, also an jenen mit tiefen Fallnormkosten. Seither stehen Spitäler mit hohen Fallnormkosten tendenziell im Ruf, ineffizient zu arbeiten. Doch ist dem auch immer so? Steckt hinter den 8 Prozent höheren Fallnormkosten (siehe Abb. 1), welche die AllKidS-Spitäler gegenüber gemischten Spitälern aufweisen, tatsächlich ein entsprechend grosses Effizienzpotential? Oder anders formuliert: Warum liegen die Fallnormkosten der reinen Kinderspitäler höher als die von gemischten Kliniken? 


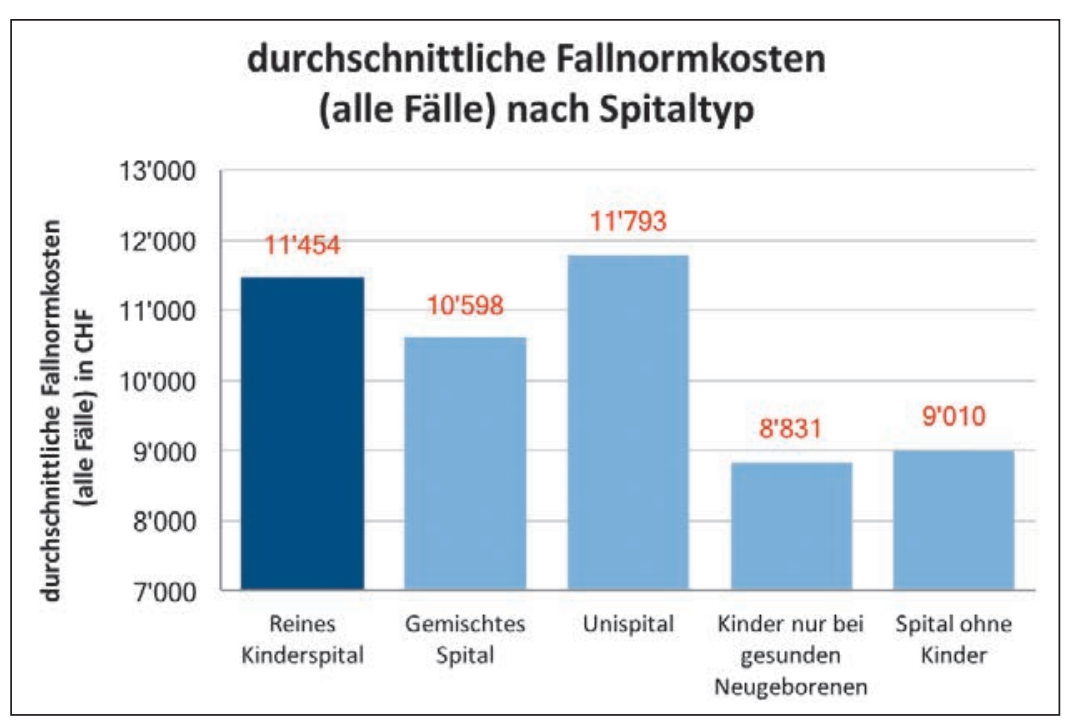

Abbildung 1: Fallnormkosten basierend auf SwissDRG-Katalog 6, bereinigte Fallnormkosten inkl. Anlagenutzungskosten. Die Kinderspitäler weisen im Vergleich zu anderen Schweizer Spitälern im Schnitt 8 Prozent höhere Fallnormkosten auf und liegen mit den Fallnormkosten näher an den Universitätsspitälern als bei gemischten Kinderspitälern. Bei den Spitälern mit nur gesunden Neugeborenen handelt es sich um Neugeborene mit der Fallpauschale P67D.

\section{Analyse zu den Mehrkosten der Kinder- spitäler unter SwissDRG}

Um diese Fragen zu klären, hat das Beratungsunternehmen Polynomics eine theoretische und empirische Analyse zu den Mehrkosten der Kinderspitäler unter SwissDRG gemacht. Die Studie erfolgte im Auftrag der SwissDRG AG in Zusammenarbeit mit AllKidS. Die Kernaussage der Studie lässt an Deutlichkeit nichts zu wünschen übrig: Die Mehrkosten der AllKidS-Spitäler sind nicht das Ergebnis von Ineffizienz, sondern die Folge spezifischer Einflussfaktoren. Die Aussage fusst auf der Überprüfung von drei Hypothesen, die wir im Weiteren näher erläutern wollen:

1. Aufwendige Kinder: Sind Kinder grundsätzlich teurer als Erwachsene, weil deren Behandlungs- und Pflegebedarf höher ist und auch die Unterhaltskosten für die Angehörigen zusätzliche Mittel erfordern?

2. Patientenselektion zulasten der Kinderspitäler: Behandeln die reinen Kinderspitäler primär besonders teure Kinder, weil sie als Endversorger analog den Unispitälern schwere bzw. schwierige Fälle nicht weiterverweisen können?

3. Aufwendiges Leistungsangebot: Ist das umfassende und hochspezialisierte Leistungsangebot der reinen Kinderspitäler der Grund ihrer Mehrkosten gegenüber gemischten Kliniken?

\section{$\mathrm{Zu}$ Hypothese 1}

Entgegen landläufiger Erwartungen sind Spitalbehandlungen von Kindern nicht teurer als die von Erwachse- nen. Im Gegenteil: Sie sind rund 20 Prozent günstiger. Das gilt auch für die reinen Kinderspitäler. Ihr Problem besteht jedoch darin, dass nur bei Kurz- und Normalliegern tiefere Kosten resultieren. Bei den Langliegern weisen Kinder hingegen 60 Prozent höhere Fallnormkosten auf als Erwachsene. Da der Anteil der Langlieger bei den spezialisierten Endversorgerspitälern für Kinder deutlich höher liegt als in gemischten Spitälern, werden die generellen Minderkosten bei Kindern dadurch nicht nur zunichtegemacht, sondern überproportional in die Höhe getrieben. Hingegen werden die Langlieger in den eigenständigen Kinderspitälern gesamthaft günstiger behandelt als in gemischten Spitälern. Von Ineffizienz kann hier also nicht die Rede sein.

\section{Zu Hypothese 2}

Neben Langliegern haben die reinen Kinderspitäler ein Problem, das der Tarifstruktur und nicht ineffizienter Arbeitsweise geschuldet ist. Die Mehrkosten entstehen durch die Zuweisung von Patienten mit einem Geburtsgebrechen (IV-Fälle), insbesondere kranker Neugeborener, und eine höhere Anzahl Diagnosen.

Eine Differenzierung zwischen gesunden Neugeborenen im Wochenbett und kranken Neugeborenen auf der Intensivstation ist über den internationalen Diagnosekatalog ICD-10 alles andere als einfach. Aber vor dem Hintergrund, dass AllKidS-Spitäler ausschliesslich kranke, d.h. aufwendige Neugeborene behandeln und dort im Unterschied zu gemischten Spitälern keine Geburten von gesunden Neugeborenen stattfinden, liegt es auf der Hand, dass für die reinen Kinderspitäler automatisch höhere Fallnormkosten entstehen. Dieses Problem ist innerhalb von SwissDRG durchaus erkannt. Aber weil die Fallzahlen für kranke Neugeborene sehr niedrig sind und diejenigen für gesunde Neugeborene und Neugeborene mit leichten Adaptationsstörungen sehr hoch, ist die korrekte tarifarische Abbildung bisher ein noch ungelöstes Problem. Auch hier kann keine Rede von Ineffizienz sein.

\section{Zu Hypothese 3}

Die unabhängigen Kinderspitäler weisen komplexere Patientenstrukturen auf als gemischte Kliniken und haben daher einen überproportionalen Aufwand zu leisten. Höhere Fallnormkosten entstehen den AllKidS-Spitälern aber nicht nur wegen des speziellen Patientenmix, sondern auch wegen des spezifischen Leistungsangebots. SwissDRG wurde primär für Normalversorger konzipiert, wo sich gegenläufige Effekte ausgleichen können. Bei den Endversorgerspitälern spielt dieser Effekt jedoch viel weniger. Die Abweichungen ihrer Fallnormkosten nach oben sind häufiger und heftiger. Ganz besonders ins Gewicht fällt dabei die bereits erwähnte Kategorie 
MDC 15. Berechnet man pro MDC für alle von den AllKidS-Spitälern angebotenen DRGs die schweizweit durchschnittlichen Fallnormkosten, zeigt sich, dass in ihrem Leistungsangebot viele davon überdurchschnittlich hoch sind. Und weil die AllKidS-Spitäler hier gegenüber gemischten Kliniken überproportional hohe Fallzahlen aufweisen, geht die Rechnung für sie unter dem Strich einfach nicht auf.

Denselben Effekt - eine hohe Abweichung nach oben gegenüber dem Mittelwert und viele Fälle - sehen wir übrigens auch bei MDC 4 (Krankheiten und Störungen der Atmungsorgane). MDC 18 (HIV sowie infektiöse und parasitäre Erkrankungen), MDC 16 (Krankheit des Blutes, der blutbildenden Organe und des Immunsystems), MDC 19 (psychische Krankheiten), MDC 23 (Faktoren, die den Gesundheitszustand beeinflussen, und andere Beanspruchungen des Gesundheitswesens) sowie MDC 22 (Verbrennungen) weisen hohe Kostenabweichungen auf, schlagen aber mengenmässig nicht so stark durch wie MDC 15 und 4. Sie zeigen jedoch, dass die AllKidS-Spitäler übermässig komplexe Fälle behandeln und diese in der Regel die Kosten als sogenannte Geburtsgebrechen bei der Invalidenversicherung belasten (siehe Studie, S. 46, Abb. 11). Die Weiterentwicklung des SwissDRG hat bisher für die spezialisierte Kinder- und Jugendmedizin keine wesentlichen Verbesserungen gebracht. So rechnet die SwissDRG AG für das Jahr 2018 mit einem Kostendeckungsgrad von 91,5 Prozent, für 2019 mit 93,7 Prozent (siehe Systempräsentation SwissDRG-Version 8.0).

\section{Sachgerechte Tarife für unsere Kinder- spitäler - stationär wie ambulant}

Angesichts dieser speziellen und bedeutsamen Rolle der hochspezialisierten Kinder- und Jugendmedizin in der Gesundheitsversorgung unseres Landes ist es an der Zeit, diesem Thema auch aus tariflicher Optik die nötige Aufmerksamkeit zu geben. Mit Blick auf die seit 2012 geltende SwissDRG-Tarifstruktur scheinen auch Grundsatzdiskussionen angebracht. Denn laut den Polynomics-Autoren liegen die eigentlichen Herausforderungen der in den Spitälern geleisteten Kinder- und Jugendmedizin nicht per se in der Umsetzung des heutigen Vergütungssystems. Wörtlich heisst es im Bericht: «Die Herausforderungen liegen vielmehr darin begründet, dass basierend auf SwissDRG ein Benchmarking mit Fallnormkosten angestrebt werden soll; ein Vorgehen, das international einmalig ist.» Klar ist, dass der Kinderund Jugendmedizin in unserem Land ohne sachgerechte Tarife eine erhebliche Schwächung droht. Um das zu verhindern, ist jedoch eine umfassende, gesamtheitliche Sicht notwendig, d.h. über den stationären Tarif
Der Stellenwert der Kinder- und Jugendmedizin im Rahmen von SwissDRG

Die Polynomics-Studie verfolgte das Ziel, mittels theoretischer und empirischer Analysen die Ursachen der Mehrkosten der AllKidS-Spitäler unter SwissDRG zu eruieren. Dass die Studie gemacht wurde, unterstreicht den Stellenwert der Kinder- und Jugendmedizin im Rahmen der Systementwicklung. Die SwissDRG AG hat zum Schlussbericht eine eigene Einschätzung vorgenommen, die auf die wichtigsten Ergebnisse aus übergeordneter Tarifsystemoptik fokussiert. Nachzulesen ist diese unter [3].

(SwissDRG) hinaus. Denn auch der ambulante Arzttarif TARMED bereitet unseren Kinderspitälern seit Jahren Sorgen. 95 Prozent der Patienten in den AllKidS-Spitälern werden ambulant behandelt (30 Prozent der Erträge). Bei einer Kostendeckung von 65 bis 70 Prozent reisst das ein grosses Loch in die Kasse. Mit dem jüngsten Tarifeingriff des Bundesrates hat sich die bereits schwierige finanzielle Situation der Kinderspitäler weiter verschärft. So dürfte das Defizit der drei AllKidS-Spitäler im ambulanten Bereich von 21 Millionen Franken (Geschäftsjahr 2016) im laufenden Jahr auf über 30 Millionen ansteigen. Dass es sich auch hier um ein Tarifproblem handelt und nicht um die Frage von mehr oder weniger Effizienz, zeigt sich daran, dass die Anzahl ambulanter Behandlungen in den letzten Jahren stark ansteigt, während die durchschnittlichen Kosten pro Behandlung tendenziell abnehmen.

Man kann es drehen und wenden, wie man will: Die wirtschaftliche Grundlage unserer spezialisierten Kinder- und Jugendmedizin gerät immer mehr unter Druck, weil die aktuellen Tarife nicht sachgerecht sind. Wohin das führt, ist klar. Die Versorgung der kranken Kinder und Jugendlichen wird mittelfristig darunter leiden. Denn nicht nur die eigenständigen Kinderspitäler sind defizitär, sondern alle Kinderkliniken, die am Ende der Versorgungskette stehen. Sie überleben nur, weil sie querfinanziert werden. Unter solchen Bedingungen werden Fortschritt und Weiterentwicklung unseres Fachgebietes massiv erschwert. Die Verlierer einer solchen Entwicklung sind am Ende die Kinder, deren Versorgung in Ermangelung spezifischer Expertise an Qualität verliert.

\section{Interessenkonflikte}

Es bestehen keine Interessenkonflikte ausser den genannten

\section{Bildnachweis}

Abbildung 1 (eigene Darstellung) gemäss «Theoretische und empirische Analyse zu den Mehrkosten der Kinderspitäler unter SwissDRG», Polynomics AG, Dr. Philippe Widmer et al., S. 6.

\section{Literatur}

1 www.swissdrg.org/application/files/8515/1325/7799/Polynomics_ Fallkostenanalyse_Kinderspitaeler_Schlussbericht.pdf

2 Annic Weyersberg, Bernd Roth, Christiane Woopen, Pädiatrie: Folgen der Ökonomisierung, Deutsches Ärzteblatt, Deutsches Ärzteblatt 2. März 2018, Jg. 115, Heft 9, S A382-6.

3 www.swissdrg.org/application/files/8715/1325/7649/Zusammenfassung__und_Schlussfolgerungen_der_SwissDRG_AG.pdf 